\title{
Despeckling of Oil Spill SAR Images using Fusion Technique
}

\author{
V.Radhika \\ Prof \& Head, Department of Computer Science, \\ Sri Krishna Arts and Science College, \\ Coimbatore-8, India.
}

\author{
Dr. G. Padmavathy \\ Prof\&Head, Department of Computer Science, \\ Avinashilingam University for Women, \\ Coimbatore-43, India
}

\begin{abstract}
Synthetic aperture radar (SAR) oil spill images are corrupted by speckle noise due to random interference of electromagnetic waves. The speckle degrades the quality of the oil spill images and makes interpretations, analysis and classifications of SAR images harder. Therefore, some speckle mitigation is necessary prior to the processing of SAR oil spill images. In this paper, some basic speckle reduction filters like Kuan, Lee, Frost, antistrophic diffusion and SRAD filters are used. A New method is proposed for despeckling of SAR oil spill images which combines the frost filter with relaxed median filter. The proposed method gives better results when compared to other methods in terms of statistical parameters like PSNR, MSE, energy and entropy value. The approach can also reduce the computing time compared with other approaches.
\end{abstract}

\section{General Terms}

Image restoration, Noise reduction, Speckle reduction algorithms.

\section{Keywords}

Kuan, Lee, Frost, anisotrophic diffusion, SRAD, PSNR, MSE, Entrophy, Relaxed median, oil spill.

\section{INTRODUCTION}

There are different types of marine pollution [1], oil is a major threat to the sea ecosystems. The source of the oil pollution can be located on the mainland or directly at sea Discharges are coming from sea-based sources like ships or offshore platforms. Marine pollution from sea-based oil sources can be accidental or intentional. Nowadays, the number of marine accidents and the volume of oil released are reducing to a greater extent. On the other side, regular tanker handling activities results in release of oily ballast water and tank washing residues. In addition to that, fuel oil sludge, engine room wastes and foul bilge water produced by ships, also settles in the sea. Due to the increase in marine transportation, also increase the oil discharges. All kinds of ships and oil tankers discharge the oil and other waste materials in the sea.

The different types of vessels, airplanes, and satellites are the tools to detect and monitor oil spills. Vessels, can cover a very limited area to detect oil at sea when equipped with specialized radars. Mainly vessels are necessary for oil sampling. The airplanes and satellites equipped with Synthetic Aperture Radar (SAR) are mainly used to monitor sea-based oil pollution. SAR is an active microwave sensor, which captures two dimensional images. The brightness of the captured image is a reflection of the properties of the target-surface. The oil spill detection using a SAR image relies on the fact that the oil on the sea decreases the backscattering of the sea surface resulting in a dark formation that contrasts with the brightness of the surrounding spill-free sea. Space borne SAR sensors are extensively used for the detecting the oil spills in the marine environment, as they are independent from sun light, they are not affected by cloudiness, they cover large areas and are more cost-effective than air patrolling. Radar backscatter values from oil spills are very similar to backscatter values from very calm sea areas and other ocean phenomena named "look-alikes" (e.g. currents, eddies).

Oil spills are also caused when there is a damage in oil pipes occurs across its cross section. Surveys [2] show that annually, $48 \%$ of the oil pollutants in the oceans are fuels, $29 \%$ are crude oils and tanker accidents contribute only $5 \%$ to this pollution. The major challenging task is to detect the presence of oil spills across the globe. Nowadays, many technical and research bodies serve this purpose. People face a number of challenges due to this problem and hence an attempt is made to have some segmentation measures in this paper.

RADAR images [7] are greatly affected by speckle noise natural phenomenon generated by the coherent processing of radar echoes. The presence of speckle noise not only reduces the interpreter's ability to resolve fine detail, but also makes automatic image segmentation more difficult. The gamma map filter is primarily used on radar data to remove high frequency noise. Thus an effective speckle filtering is required as a preprocessing step before applying any of these image processing techniques. The objective of any speckle filtering is

(1) To effectively suppress the multiplicative noise in homogenous regions,

(2) To preserve and enhance edges and image details, and

(3) To provide a visually good appearance for detection of oil spills from SAR images.

Usually these requirements can hardly be met simultaneously.

Synthetic Aperture Radar (SAR) is used for the oil spill detection. It was a space borne technique where radars captured the images of the oil spills and an analysis of it were made to differentiate the oil spills with its "look-alikes". The "lookalikes" includes natural slicks, grease ice, threshold pipelines and passing vessels that were visualized exactly like an oil spill on a homogenous background. The basic functionality of SAR included capturing of images by the radars on backscattering of rays by the water bodies. Oil usually absorbs heat during days 
and in nights they dissipate the heat into the water that they appear colder than waters at night. So even the thinnest layer of oil spilling can be detected. Firstly, the radars capture the images of the water bodies and then analyzed. Careful analysis of the image is done in the regions where dark spots are found before that preprocessing is necessary. In this paper despeckling of oil spill images is explained by fusion method.

Many of the despeckling filters currently available (Lee, Kuan, and Frost etc.) [5-28] operate by smoothing over a fixed window. All these filters named statistic filters are based on the statistical characteristics of the SAR image and the speckle. The results indicate that the statistical filters can reduce the speckle better, but the detail of the image is lost greatly, and the normal hybrid filters can keep the detail well but the effect of reducing the speckle is not good. From this viewpoint, to make use of the advantages of the both filters, a Fusion method is proposed. In this paper, a combination of Frost speckle reduction filter and Relaxed Median filter is proposed for SAR oil spill image denoising. And at the end of the paper, the experimental results show that this new method can reduce the speckle to great extend and also maintain the image detail better.

This paper is organized as follows: Section II discusses the speckle noise model need for Speckle noise filtering in SAR images and the related work. Section III presents the novel method to despeckling algorithm for SAR images. The simulation results based on different parameter evaluations are presented in section IV. Finally, conclusions are given in section V.

\section{SAR SPECKLED MODEL AND NEED FOR SPECKLE FILTERING}

\subsection{SAR speckled image model}

It is well established that speckle manifests in the form of multiplicative noise, which expresses the observed intensity as the product of the scene signal intensity and speckle noise intensity.

Let $z_{i j}$ be the degraded pixel of an observed image and $x_{i j}$ be the noise-free image pixel to be recovered. With the multiplicative noise model,

$$
Z_{i j}=x_{i j} u_{i j}
$$

in which $u_{i j}$ represents the multiplicative noise with unit mean and standard deviation $\sigma_{u}$. Speckle noise (Z) is often modeled as a multiplicative noise over a noise-free image $X$, where $Z$ is supposed to be uncorrelated to $X$ [24]. The model follows the expression:

$$
\mathrm{Y}=\mathrm{X} \cdot \mathrm{Z}
$$

where $\mathrm{Z}$ has an unitary mean with variance $\sigma_{z}^{2}$. As $\mathrm{Z}$ and $\mathrm{X}$ are assumed to be statistically independent, the sample mean and the variance over homogeneous areas, i.e. areas of constant average intensity, can be derived from the expressions [19]:

$$
\begin{aligned}
& \bar{Y}=\bar{X} \cdot \bar{Z}=\bar{X} \\
& \sigma_{2}=\frac{\sigma_{y}}{\bar{X}}
\end{aligned}
$$

The speckle noise standard deviation $\left(\sigma_{z}\right)$ provides the amount of variation of terrain reflectivity in SAR images for regions where the multiplicative model holds. Speckle presents a well known statistical model for homogeneous regions, which can be quantified using Eq. 4 .

$$
\beta_{z}=\frac{0.5227}{\sqrt{L}}
$$

The variation coefficient $\left(\sigma_{z}\right)$ represents the speckle noise fluctuations in SAR images, calculated according to Eq.4, which can be used as a measure of homogeneity. For homogeneous regions, $\left(\sigma_{z}=\beta_{z}\right)$ and the sample mean, $\left(\boldsymbol{\mu}_{y}\right.$, and standard deviation, $\sigma_{y}$ can be estimated over a window comprising W pixels in these homogeneous regions.

\subsection{Need for Speckle filtering}

Speckle arises because the resolution of the sensor is not sufficient to resolve individual scatters within a resolution cell. Speckle is a large problem in oil spill SAR images since even a homogeneous area has a statistical distribution with large standard deviation. Incoherent averaging multiple looks may reduce speckle (Leberl, 1990). Another possibility is to smooth the speckles after the image has been formed. Barni et al. (1995) tested two types of filters, general noise reducing filters that do not assume any a priori speckle model and adaptive filters assuming a multiplicative speckle model (speckle noise by a multiplicative model is described by Lee (1981)). Better results were reported for the latter type in their oil spill detection algorithm. For oil spill applications, a filter should suppress speckle noise, but still preserve small and thin oil spills.

\subsection{Related work}

The speckle in oil spill SAR images is often considered as undesirable and several noise removal filters have been proposed. Unlike the additive white Gaussian noise model adopted in most denoising methods, oil spill SAR imaging requires specific filters due to the signal dependent nature of the speckle intensity. In this section, a classification of standard adaptive filters and methods for speckle reduction are presented.

The adaptive filters are widely used in image restoration because they are easy to implement and control. The commonly used adaptive filters - the Lee's filter [6], Frost's filter [7], and Kuan's filter [8] -assume that speckle noise is essentially a multiplicative noise. Many changes in these classical filters have been proposed since to get better results.In the 1990s, Lopes et al. [5] improved the Lee's and Frost's filters by classifying the pixels in order to apply specific processing to the different classes. Based on this idea, many Adaptive Speckle Reduction filter (ASR) exploits local image statistics to determine specific 
areas to be processed further. In [8], the kernel of the adaptive filter is fitted to homogeneous regions according to local image statistics. Analyzing local homogeneous regions was also investigated in [9], [10] to spatially adapt the filter parameters. Note that the Median filter has been also examined for speckle reduction in [9].

Adapted formulations of the Anisotropic Diffusion filter (AD) [12] and the Total Variation minimization scheme (TV) [13] have been developed for US imaging. In [14] and [18], the Speckle Reducing Anisotropic Diffusion (SRAD) was introduced and involves a noise-dependent instantaneous coefficient of variation. In [15] the Nonlinear Coherent Diffusion (NCD) filter is based on the assumption that the multiplicative speckle in US signals is transformed into an additive Gaussian noise in Log-compressed images. Recently, the Oriented SRAD (OSRAD) filter has been proposed in [16]; this filter takes into account the local directional variance of the image intensity, i.e., the local image geometry. Finally, the TV minimization scheme has been adapted to ultrasound imaging in [18] to [20]. Unlike the previous adaptive speckle filters, all the considered PDE-based approaches are iterative and produce smooth images while preserving edges. Nevertheless, meaningful structural details are unfortunately removed during iterations.

Several conventional wavelet thresholding methods [21]-[23] have also been investigated for speckle reduction with the assumption that the logarithm compression of images transforms the speckle into an additive Gaussian noise. The aforementioned approaches can be also combined in order to take advantage of the different paradigms. In [23], the image is preprocessed by an adaptive filter in order to decompose the image into two components. A Donoho's soft thresholding method is then performed on each component. Finally, the two processed components are combined to reduce speckle. PDE-based approaches and a wavelet transform have been also combined as proposed in [24].

\section{FUSION ALGORITHM FOR DESPECKLING OIL SPILL SAR IMAGES}

Fusion algorithm is a class of non-linear filters which consists of two stages. The first stage consists of a frost speckle reduction filter and the second stage is a relaxed median filter, which processes the output of frost speckle reduction filter. The model enjoys the benefit of both linear frost speckle reduction filter and relaxed median filter.

This paper explains a new hybrid filter method, based on frost speckle reduction filter and relaxed median filter. First, a preliminary filtering based on the basic despeckle method is first carried out to coarsely removal of speckle noise in oil spill images using frost filter. This is followed by a fine smoothing using a relaxed median filter based method for object pixel as its inputs. The algorithm are explained below

$\begin{array}{ll}\text { (i) } & \text { Frost speckle reduction filter } \\ \text { (ii) } & \text { Relaxed median method } \\ \text { (iii) } & \text { Proposed Fusion filter }\end{array}$

\subsection{Frost speckle reduction filter}

The Frost [7] filter is an adaptive radar filter that uses the local image statistics in the filtering process, assuming a negative exponential distribution for the speckle noise. The filter performs a weighted average of the cell values in the filter window, with the weights for each cell being determined from the local statistics to minimize the mean square error of the signal estimate. The filter weight for a cell is a negative exponential function of the noise standard deviation (calculated locally for each filter window) and also decreases with distance from the center cell. The center cells are weighted more heavily as the variance in the filter window increases. The filter therefore smoothes more in homogeneous areas, but provides a signal estimate closer to the observed value of the center cell in heterogeneous areas. The Frost filter has no user-defined parameters.

The mathematics behind Frost filter is given below. A Frost filter adapts to the noise variance within the filter window by applying exponentially weighting factors $\mathrm{M}$ as given in Equation (6). These weighting factors decrease as the variance within the filter windows reduces.

$$
M n=\exp \left(-\left(D A M P *(S / I M)^{\wedge} 2\right) * T\right)
$$

In the Equation, DAMP is a factor that determines the extent of the exponential damping for the image. The larger the damping value, the heavier is the damping effect. Typically the value is set to $1 . \mathrm{S}$ is the standard deviation of the filter window, $\mathrm{Im}$ is the mean value within the window and $\mathrm{T}$ is the absolute value of the pixel distance between the center pixels to its surrounding pixels in the filter window. The value of the filtered pixel is replaced with a value calculated from weighted sum of each pixel value $\mathrm{Pn}$ and the weights of each pixel $\mathrm{Mn}$ in the filter window over the total weighted value of the image as given in Equation ( 7).

$$
\operatorname{Img}(i, j)=\operatorname{SPn} * \frac{M n}{S M n} \ldots \ldots(7)
$$

The parameters in the Frost filter are adjusted according to the local variance in each area. Low variances causes extensive smoothing and high variance, smoothing is normal and edges are also retained.

It can also be observed that LEE filter and frost filters perform the worst for curved structures where decision making becomes a complex one. Hence with a mask size of $3 \times 3$ for speckle detection and suppression.

The Frost filter differs from the Lee and Kuan filters with respect that the scene reflectivity is estimated by convolving the observed image with the impulse response of the coherent imaging system. The system's impulse response has been calculated by minimizing the mean square error between the observed image and the scene reflectivity model which is assumed to be an autoregressive process. These filters have achieved a degree of suppressing speckle while preserving the texture information

\subsection{Relaxed median filter}

The output of the relaxed[24][25] median (RM ) filter with parameters $\ell$ and $\vartheta$ is given by 
$y_{i}=R M_{\ell \vartheta}\left(w_{i}\right)=\left\{\begin{array}{l}\left.x_{i}, \text { if } x_{i} \in\left[\left[w_{i}\right]\right]_{\ell},\left[w_{i}\right]_{\vartheta}\right] \\ w_{i}, \text { otherwise }\end{array}\right.$

where $\ell$ and $\vartheta$ are such that $1 \leq \ell \leq N+1 \leq 2 N+1$. note when $\ell=1$ and $\vartheta=2 N+1$, the $R M_{\ell \vartheta}$, the identity filter (no filtering), and when $\ell=1, \vartheta=N+1$, the output of the $R M_{\ell}$ is simply the median. Fig. 1 illustrates the structure of the relaxed median filter. It is worth noting that the symmetric relaxed median filter is also called rank conditioned median filter [5]. In [6], the output distribution of the relaxed median filter is given in a more generalized form, and its statistical properties for details preservation are also presented and illustrated.

\section{Properties of Relaxed median filter}

In this subsection, some deterministic properties of relaxed median[24] filters are presented. First, it is easy to show that relaxed median filters are translation and scale invariant, that is

$$
\begin{gathered}
R M_{l u}\left(s W_{i}\right)=\operatorname{sRM}_{l u}\left(W_{i}\right)+t, \forall_{s}, t \\
\in R \ldots \ldots \ldots(9)
\end{gathered}
$$

For the sake of simplicity, we consider a symmetric relaxed median filter, that is, the parameters $\ell$ and $\vartheta$ are symmetric This restriction is appropriate when the signal and noise are symmetric $\vartheta=2 N+2-\ell$ and to ensure the unbiasedness of the filter. Therefore, the symmetric relaxed median $R M(2 N+1 ; \ell)$ filter is completely specified by two parameters: the window width and the lower bound $\ell$. The deterministic behavior of the relaxed median filter can be analyzed by considering its effect on arbitrary sequences.

\subsection{Proposed Fusion Filter}

The discrete form of frost filter

$$
M n=\exp \left(-\left(D A M P *(S / I M)^{\wedge} 2\right) * T\right)
$$

Relaxed median filter can be used in combination with above equation to remove large spike noises. The proposed hybrid method is defined as follows:

$$
\vartheta_{\ell \vartheta}\left(w_{i}\right)=R M_{\ell \vartheta}\left(M_{N_{\ell \vartheta}}-i m g_{\ell \vartheta}\right)
$$

where RM means Relaxed Median filter with lower bound $\ell$ and upper bound $\vartheta$.

\section{EXPERIMENTAL RESULTS}

To test the accuracy of the filtering algorithms, four steps are followed.

i) First, an uncorrupted oil spill image is taken as input. ii) Second speckle noise is added to an oil spill image.

iii) Third, filtering algorithm is applied for noise added image.

iv) Fourth, the performance evaluation is obtained by the statistical method like Energy, Entropy, PSNR and MSE.

The reconstruction of an image has the dimensions of 256 pixel intensity. The oil spill [29] images in this contain a wide variety of subject matters and textures. Most of the images used are oil spill slick images with defect and without defect images. The Energy, Evaluation time and MSE must be less value for a better filtering algorithm. The PSNR value must be high for a better filtering algorithm.

\section{a.) Energy}

The gray level energy indicates how the gray levels are distributed. It is formulated as,

$$
E(x)=\sum_{i=1}^{x} p(x)
$$

where $\mathrm{E}(\mathrm{x})$ represents the gray level energy with 256 bins and $\mathrm{p}(\mathrm{i})$ refers to the probability distribution functions, which contains the histogram counts. The energy reaches its maximum value of 1 when an image has a constant gray level.

The larger energy value corresponds to the lower number of gray levels, which means simple. The smaller energy corresponds to the higher number of gray levels, which means complex.

\section{b.) Entropy}

Suppose that two discrete probability distributions of the images have the probability functions of $\mathrm{p}$ and $\mathrm{q}$, the relative entropy of $\mathrm{p}$ with respect to $\mathrm{q}$ is then defined as the summation of all possible states of the system, which is formulated as,

$d=\sum_{i=1}^{k} p(i) \log _{2} \frac{p(i)}{q(i)}$

\section{c.) Mean Square Error (MSE)}

The metric MSE is defined as:

$M S E=\frac{1}{M N} \sum_{i=1}^{M} \sum_{i=1}^{N}[g(i, j)-f(i, j)]^{2}$

For two $m \times n$ monochrome images $I$ and $K$, one of the images is considered a noisy approximation of the other. Other metrics like RMSE, MAE and PSNR are defined using MSE.

\section{d.) Peak Signal to Noise Ratio (PSNR)}

Peak signal-to-noise ratio (PSNR) is used to give a quantitative evaluation. The PSNR value is calculated between the original image and the edge detected noisy images. The PSNR is most 
commonly used as a measure of quality of reconstruction of lossy compression (e.g., for image compression). A higher PSNR would normally indicate that the reconstruction is of higher quality. PSNR is calculated using formula:

$$
P S N R=10 \log _{10}\left(\frac{255^{2}}{M S E}\right)
$$

The experiments are conducted using mat lab 7.1 version. The experimental results are proved by subjective and objective method. The following table 1 shows the performance evaluation of speckle reduction filters for the average of 10 oil spill images. From the table, the proposed hybrid filter gives most suitable results based on PSNR, MSE, Energy and Entropy value.

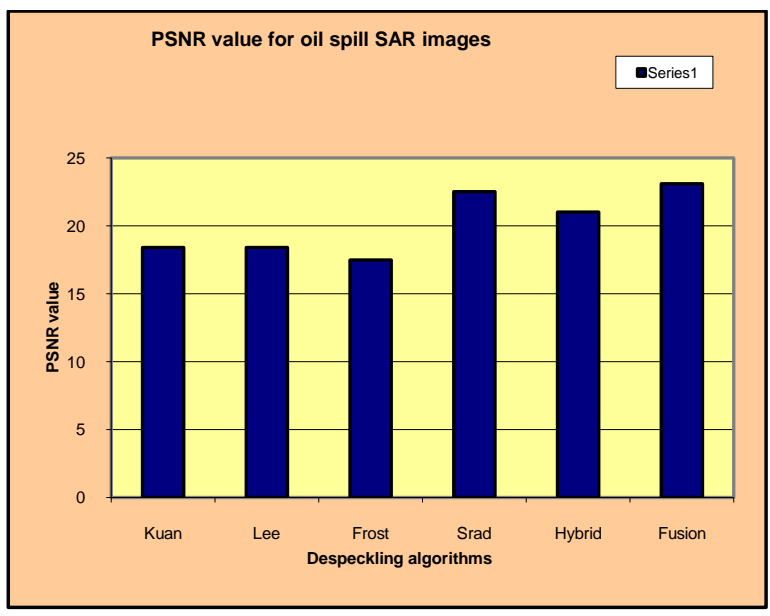

Figure(1)- PSNR value for Oil spill Images

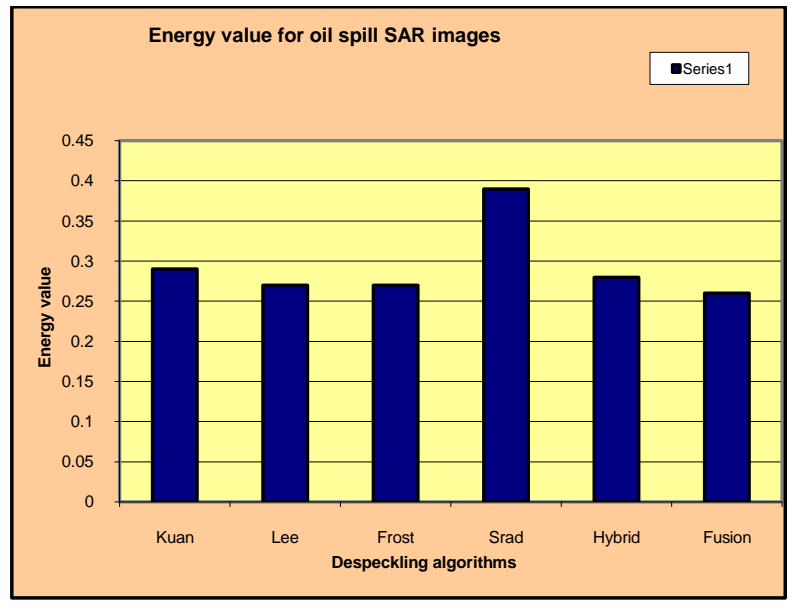

Figure(3)- Energy value for Oil spill Images

The figure (1)- (4) gives the graphical representation of PSNR, MSE, Energy, and Entropy. From the above figures it can be noted that the proposed Fusion technique gives most reliable results when compared to other speckle reduction filters.
Table. 1 Performance evaluation for speckle reduction filters

\begin{tabular}{|l|l|l|l|l|}
\hline Filter & PSNR & MSE & Energy & Entropy \\
\hline Kuan & 18.41 & 996 & 0.29 & 0.905 \\
\hline Lee & 18.42 & 954 & 0.27 & 0.906 \\
\hline Frost & 17.5 & 1021 & 0.27 & 0.97 \\
\hline Srad & 22.5 & 373.7 & 0.39 & 1.05 \\
\hline Hybrid & 21 & 441.3 & 0.28 & 1.07 \\
\hline Fusion & 23.11 & 328.6 & 0.26 & 1.09 \\
\hline
\end{tabular}

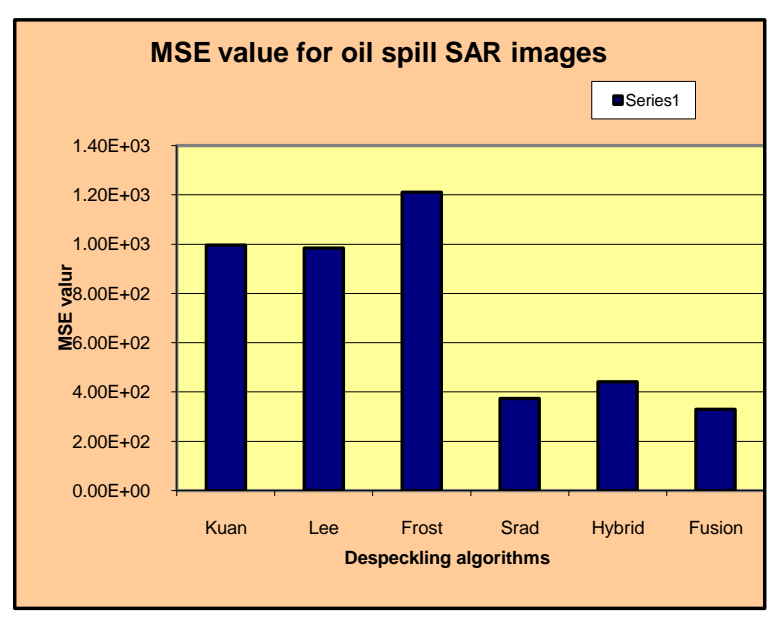

Figure(2)- MSE value for Oil spill Images

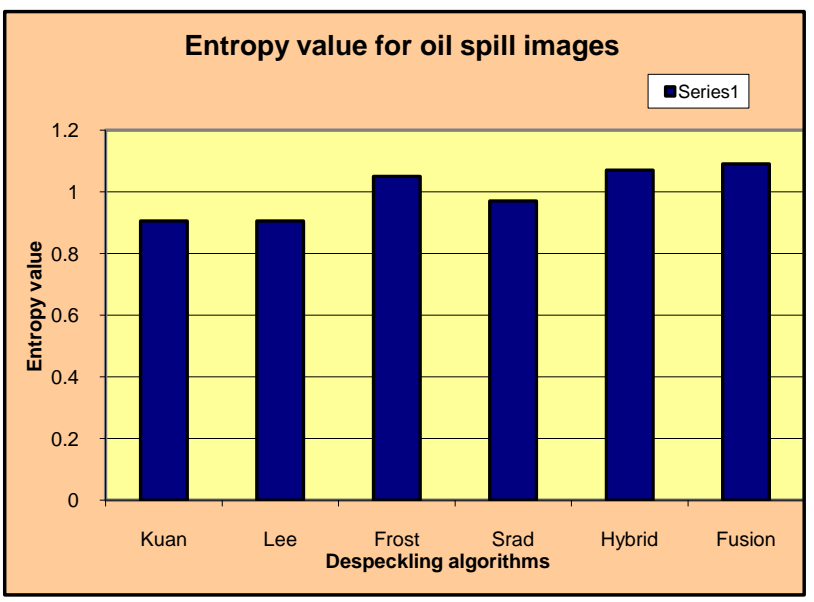

Figure(4)- Entropy value for Oil spill Images

The following figure (7) gives the pictorial representation by subjective method. By the subjective method also the proposed hybrid method gives reliable results compared to other speckle reduction technique. 


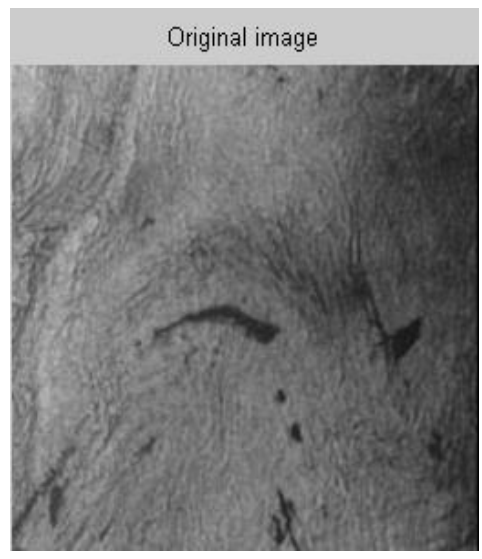

(a)

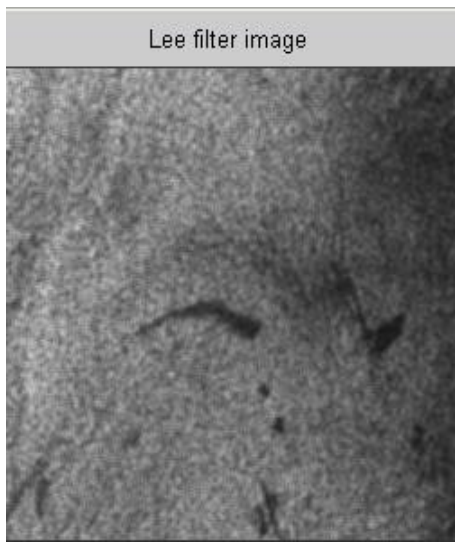

(d)

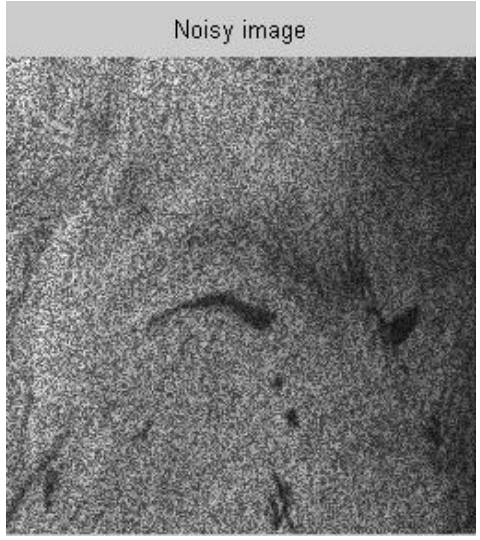

(b)

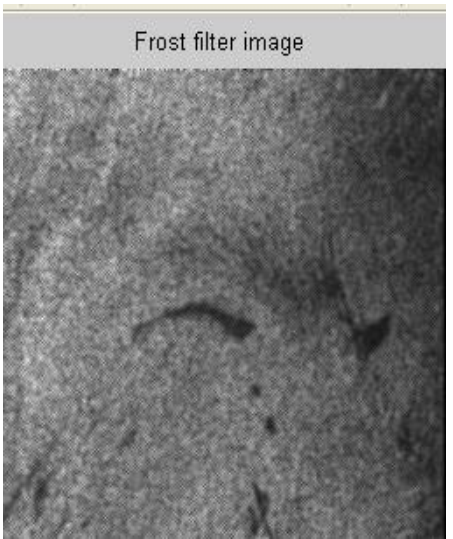

(e)

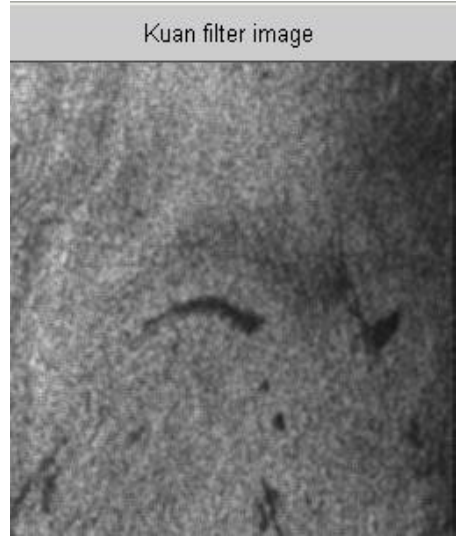

(c)

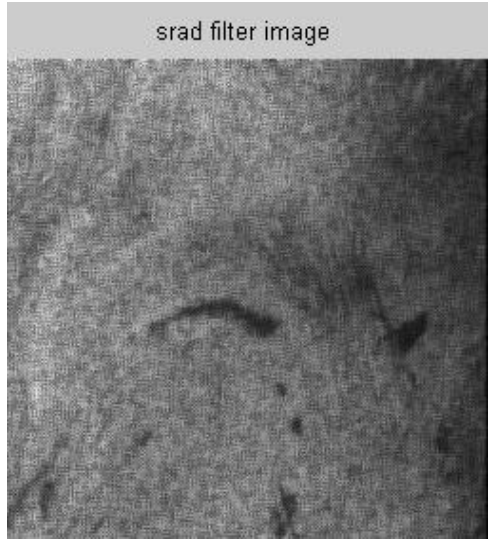

(f)

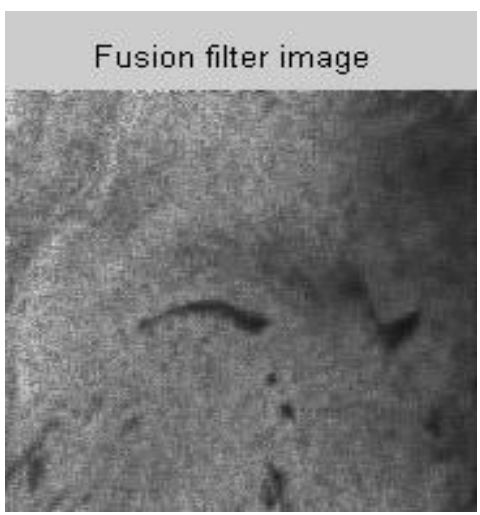

(g)

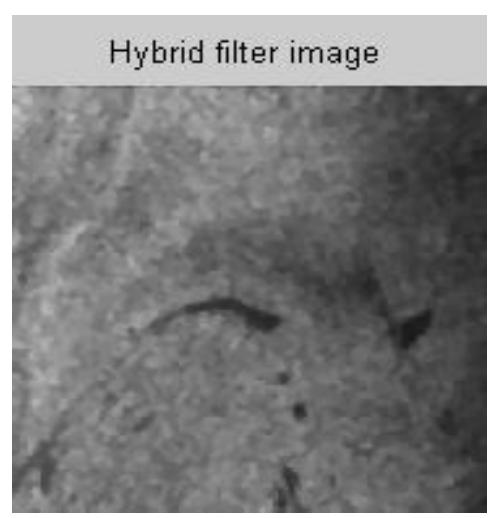

(h)

Figure (7), (a) the raw oil spill image, (b) speckle noise image, (c) kuan filtered image, (d) Lee filtered image, (e) frost filtered image, (f) srad filtered image, (g) fusion image,(h) hybrid filtered image

The figures (a-h) show the original raw oil spill images and the speckle noise applied image. Then the Kuan, Lee, Frost, SRAD and the proposed algorithm applied images are presented.

\section{CONCLUSION}

Oil spill images are corrupted by speckle noise due to random interference of electromagnetic waves. In this paper a fusion method is proposed for despeckling oil spill images which combines frost speckle reduction method and relaxed median 
filter. The Proposed method gives better results when compared to the other methods in terms of statistical parameters like PSNR, MSE, Energy and entropy value. This method also preserves the image quality without any changes in the edge, curve etc.

\section{ACKNOWLEDGMENTS}

Our thanks to the experts who have contributed towards development of the template.

\section{REFERENCES}

[1] Konstantinos N. Topouzelis ,"Oil Spill Detection by SAR Images: Dark Formation Detection,Feature Extraction and Classification Algorithms", Sensors 2008.

[2] HANG Le Minh, DUONG Nguyen Dinh, Vietnam “ Oil Spill Detection and Classification by ALOS PALSAR at Vietnam East Sea" 7th FIG Regional Conference Spatial Data Serving People: Land Governance and the Environment - Building the Capacity Hanoi, Vietnam, 1922 October 2009

[3] Mansor, H. Assilzadeh, H.M. Ibrahim, A. R. Mohamd. "Oil Spill Detection and Monitoring from Satellite Images "(C) GISdevelopment.net..

[4] Guozhong Chen Xingzhao Liu Dept. of Electron. Eng., Shanghai Jiao Tong Univ., China "An improved waveletbased method for SAR images denoising using data fusion technique" RADAR, IEEE conference 2006.

[5] T. Loupas, W. McDicken, and P. Allan, "An adaptive weighted median filter for speckle suppression in medical ultrasound image," IEEE Trans. Circuits Syst., vol. 36, pp. 129-135, 1989.

[6] J. S. Lee, "Digital image enhancement and noise filtering by use of local statistics," IEEE Trans. Pattern Anal. Mach. Intell., vol. PAMI-2, pp. 165-168, 1980.

[7] V. Frost, J. Stiles, K. Shanmugan, and J. Holtzman, "A model for radar images and its application to adaptive digital filtering of multiplicative noise," IEEE Trans. Pattern Anal. Mach. Intell., vol. PAMI-2, pp.157-65, 1982.

[8] D. Kuan, A. Sawchuck, T. Strand, and P. Chavel, "Adaptive noise smoothing filter for images with signaldependent noise," IEEE Trans. Pattern Anal. Mach. Intell., vol. PAMI-7, no. 2, pp. 165-177, Feb

[9] A. Lopes, R. Touzi, and E. Nezry, "Adaptive speckle filters and scene heterogeneity," IEEE Trans. Geosci. Remote Sens., vol. 28, pp. 992-1000, 1990.

[10] M. Karaman, M. A. Kutay, and G. Bozdagi, "An adaptive speckle suppression filter for medical ultrasonic imaging," IEEE Trans. Med. Imag., vol. 14, pp. 283-292, 1995

[11] Gagnon, L. and A. Jouan, 1997, "Speckle Filtering of SAR Images - a Comparative Study between Complex-WaveletBased and Standard Filters," in Proceedings of SPIE Wavelet Applications in Signal And Image Processing V, San Diego, CA, 80-91.

[12] Goodman, J. W., 1976, "Some Fundamental Properties of Speckle," Journal of the Optical Society of America, 66(11):1145-1150.
[13] Hagg, W. and M. Sties, 1996, "The Epos Speckle Filter: A Comparison with Some Well-Known Speckle Reduction Techniques," in Proceedings of the XVIII ISPRS Congress, July 9-19, 1996, Vienna, Austria, 135-140.

[14] Kuan, D. T., Sawchuk, A. A., Strand, T. C., and P. Chavel, 1987, "Adaptive Restoration of Images with Speckle," IEEE Transactions on Acoustics, Speech, and Signal Processing, 35(3):373-383.

[15] J. S. Lee, A simple speckle smoothing filter for signaldependent noise. IEEE Trans.On System, Man and Cybernatics, Vol. 13, No. 1, 1983, 85-89.

[16] Sheng Guofang, Hu Xin and Jiao Licheng, SAR image denoising based on data fusion. Fifth International Conference on Computational Intelligence and Multimedia Applications, ICCIMA 2003, 27-30 Sept. 2003, 143 - 148.

[17] Y. Yu and S. T. Acton, "Speckle reducing anisotropic diffusion," IEEE Trans. Image Process., vol. 11, pp. 1260 1270, 2002.

[18] K. Z. Abd-Elmoniem, A. B. Youssef, and Y. M. Kadah, "Real-time speckle reduction and coherence enhancement in ultrasound imaging via nonlinear anisotropic diffusion," IEEE Trans. Biomed. Eng., vol. 49, pp. 997-1014, 2002.

[19] K. Krissian, C. F. Westin, R. Kikinis, and K. G. Vosburgh, "Oriented speckle reducing anisotropic diffusion," IEEE Trans. Image Process., vol. 16, pp. 1412-1424, 2007.

[20] C. Sheng, Y. Xin, Y. Liping, and S. Kun, "Total variationbased speckle reduction using multi-grid algorithm for ultrasound images," in Proc. Int. Conf. Image Analysis and Processing, 2005, vol. 3617, pp. 245-252.

[21] D. Donoho and I. Johnstone, "Ideal spatial adaptation by wavelet shrinkage," Biometrika, vol. 81, no. 3, pp. 425455, 1994.

[22] R. Coifman and D. Donoho, "Translation invariant denoising," in Lecture Notes in Statistics: Wavelets and Statistics. New York: LCNS, 1995, pp. 125-150.

[23] J. E. Odegard, H. Guo, M. Lang, C. S. Burrus, R. O. Wells, L. M. Novak, and M. Hiett, "Wavelet based SAR speckle reduction and image compression," in Proc. SPIE

[24] Abdessamad Ben Hamza, “ Some Properties of Relaxed median filter”, IEEE DSP 97 , 1977, 957-960.

[25] Jeny Rajan, K. Kannan and M.R. Kaimal, ” An Improved Hybrid Model for Molecular Image Denoising", Journal of Mathematical Imaging and Vision, Vol. 31 No.1 2008, 7379.

[26] Jingfeng xiao, jing li, a. Moody A detail-preserving and flexible adaptive filter for speckle suppression in SAR imagery, International journal of Remote Sensing 2003, Vol. 24,No. 12, 2451-2465

[27] Lopez-Martinez, C. And Fabregas, X. Model based polarimetirc SAR Speckle Filter IEEE Transactions On Geoscience And Remote Sensing ,46(11):3894-3907, 2008

[28] Oil spill monitoring by remote sensing, "Database of the satellite SAR images" at http://cearac.poi.dvo.ru/en/db

[29] "Oil spill detection by satellite remote sensing" Camilla Brekke, Anne H.S. Solberg. 